\title{
Vultures and sky burials on the Qinghai-Tibet
}

\section{Plateau}

\section{Roller MaMing $^{1 *}$, Li Lee $^{1}$, Xiaomin Yang ${ }^{1}$ and Paul Buzzard ${ }^{2}$}

${ }^{1}$ Xinjiang Institute of Ecology and Geography, Chinese Academy of Sciences, No 818 Beijing Road, Urumqi, 830011, Xinjiang, P. R. of China.

${ }^{2}$ Detroit Zoological Society, 8450 W. 10 Mile Rd., Royal Oak, MI 48067, USA

*Corresponding author: maming3211@yahoo.com

http://dx.doi.org/10.4314/vulnew.v71i1.2

\section{Introduction}

In the Tibet Autonomous Region, the tradition and custom of sky burial is known as jhator meaning "giving alms to the birds" (Van Dooren 2011), and vultures are an important part of these funerals. In places where there are several jhator offerings each day, the birds sometimes must be coaxed to eat, which may be accomplished with a ritual dance. It is considered a bad omen if the vultures will not eat or if even a small portion of the body remains after the birds fly away.

The practice of jhator remains mysterious, however, and it is not well understood. For example, most people think the main species involved with jhator is the Cinereous Vulture Aegypius monachus (Anon. 2005), but according to our recent investigations, Cinereous Vultures 
and Bearded Vultures Gypaetus barbatus seldom appear at the sky burial platform. Instead, the Himalayan Vulture Gyps himalayensis is the most common participant at sky burials. Given the threats to Asian vultures, it is important to know more about this traditional practice and any effects on vulture conservation.

\section{Distribution and population}

Sky burial is a traditional funeral of the Mongolian and Tibetan ethnic minorities, and it has a long history over thousands of years in China (MaMing et al. 2016). Other ethnic minorities also keep the custom in Qinghai, Tibet, Gansu, Sichuan, Yunnan, Xinjiang and Inner Mongolia. In the past, sky burials were limited to high lamas and other dignitaries, arguably due to the relative absence of timber resources for cremation in the barren, rocky and ice-covered Himalayas, and corpses were offered to vultures after religious ceremonies (Martin 1996). Now, sky burials have been increasingly used by commoners.

Recently, the Raptor Team of the China Ornithological Society in the Yushu Tibetan Autonomous Prefecture, Qinghai Province, investigated the relationship between vultures and the custom of sky burials. We found that sky burials are held in the morning from $08 \mathrm{~h} 00$ to $11 \mathrm{~h} 00$ (Beijing time) and we observed more than 240 Himalayan Vultures consuming bodies $(\mathrm{n}=3)$ over 2.5 hours. The hair is removed from the head and is burned along with some of the debris; big bones are broken by sky burial masters (Figure 1). The bodies disappeared in less than 40 minutes. Then, as if the sky burial had never happened, all returns to peace and calm on the hill. Tibetans believe that at this point, life has completely left the body and the body contains nothing more than simple flesh. 


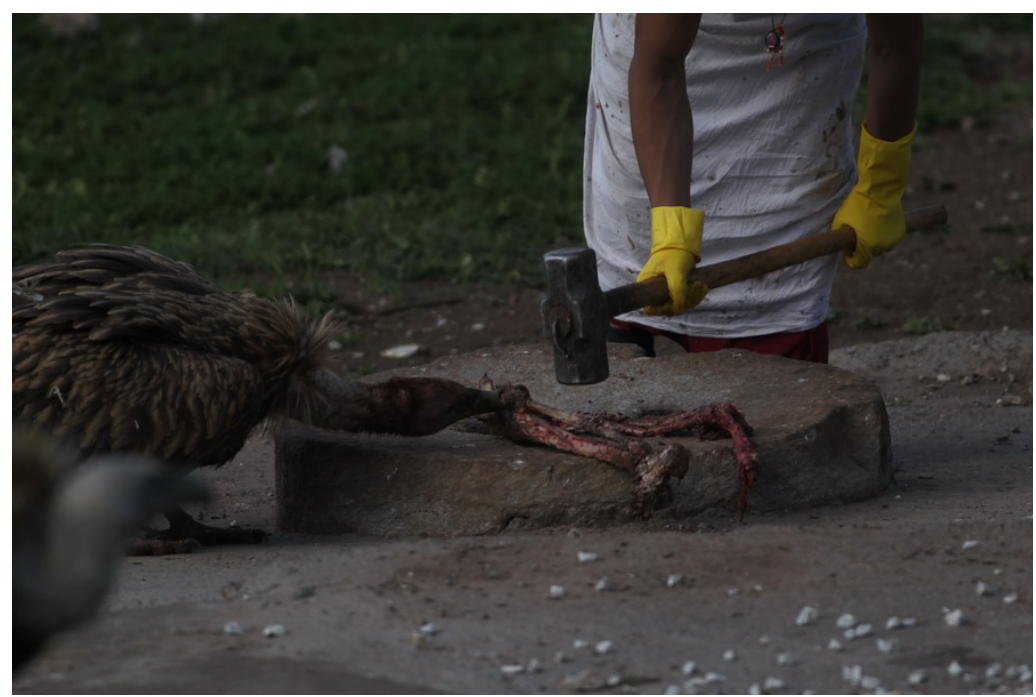

Figure 1: Big bones are broken for vultures by the sky burial master. A sky burial site in Qinghai (Photo by MaMing)

A similar situation has been recorded monks and local people. in southern Tibet at the Drigung Thel Monastery (Liu et al. 2013), where

\section{A snack or main food?} vultures occupy alpine meadows in the day and roost on the upper cliffs at night, between elevations of 4200 $5000 \mathrm{~m}$. The number of Himalayan Vultures around the monastery was estimated at 230 in 2003, 250 in 2009 and 200 in 2012 (Lui et al. 2013) This population is relatively stable, most probably due to In a temple of Ruoergai County, Sichuan Province, some vulture specimens were enshrined and hanging from the wall (Figure 2). Whilst it can be difficult for people to understand the Tibetan reverence for vultures (Zhang 2001), our aim was to determine how much of the protection of the birds by Buddhist vultures' diet comes from sky 
burials; are they a 'snack' or do they provide a major source of food for vultures? We estimated that a vulture eats 200-300 grams of meat or offal during a sky burial. However, given that the vultures stayed at the sky burial site for a long time afterwards and appeared reluctant to leave, it is possible they may not have enough food. Given the number of sky burials, it seems unlikely that human corpses provide only $2 \%$ of the amount of food in the Tibetan plateau, as has been suggested elsewhere (Lu et al. 2009).

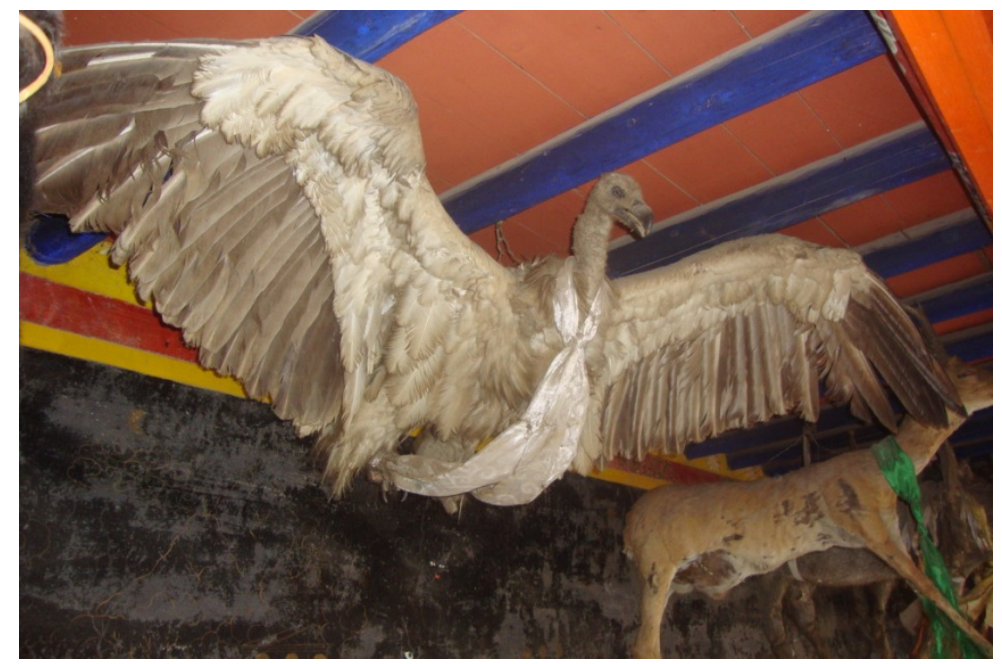

Figure 2: In Ruoergai County, Sichuan Province, vultures samples were suspended in the Daza Temple (photo by MaMing)

To better understand the relationship people choose a sky burial, at a between the Tibetan population and human mortality rate of $7-9 \%$, there sky burials, there are 6.28 million would potentially be a large number Tibetan people (National Bureau of of corpses available to vultures. Statistics 2010). If $80 \%$ of these According to public data (Xinhua 
2013), a total of 2000 sky burial sites were distributed over the Tibetan plateau receiving 50,000 corpses per year; this could feed approximately 20,000 vultures. It is therefore possible that sky burials contributed almost 20\% of the total food for Tibetan vultures, which is a far higher proportion than the $2 \%$ suggested by Lu et al. (2009).

There is some connection between the number of corpses and the number of vultures (Table 1). In July 2016, we observed and visited for five days in Yushu, where we found that the number of vultures was related to the number of corpses, with many vultures clustering around the burial station. When there are more funeral corpses, there are more vultures. We recorded many aspects of the sky burial with pictures in Tibet, Sichuan and Qinghai (see Appendix figures), such as fighting, dancing, tug of war, sunbathing, etc. There were also wild dogs and Tibetan mastiffs present, which ate the bones at a site (Figure 3). For Tibetan Buddhists, sky burial is the template of instructional teaching on the impermanence of life, the resolution of grief in the survivors is intertwined with the journey to rebirth of the deceased (Goss and Klass 1997). 


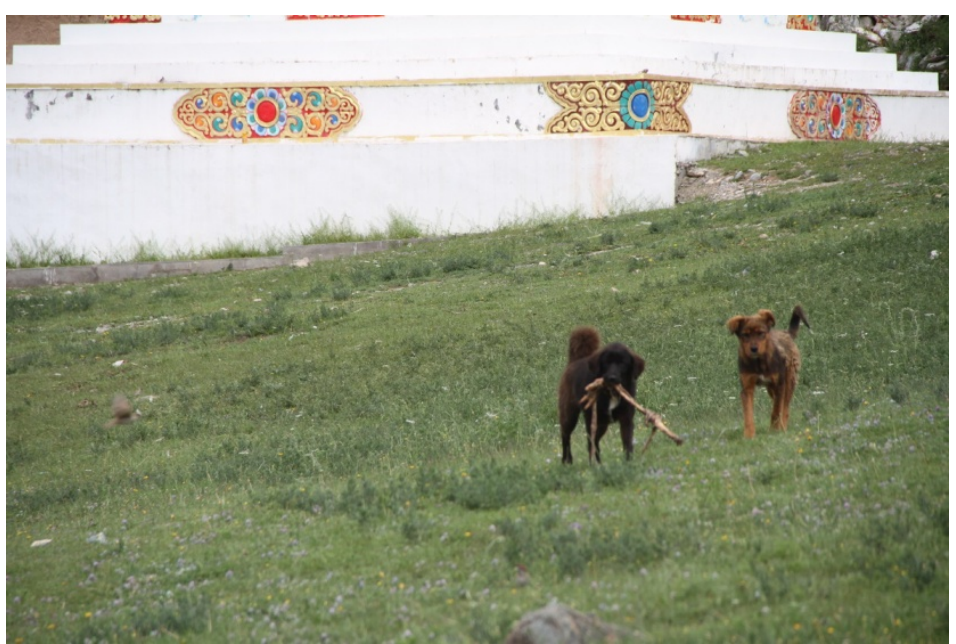

Figure 3: Wild dog with an arm from a sky burial in Qinghai (Photo by Lee)

Table 1: Observation records of sky burials with vulture numbers in Yushu Tibetan Autonomous Prefecture

\begin{tabular}{l|l|l|l|l}
\hline Date & Burial time & $\begin{array}{l}\text { Number of } \\
\text { corpses }\end{array}$ & Vulture number & Comments \\
\hline 14 July & $8: 00-11: 00$ & 3 (c. 120kg) & 240 & \multicolumn{2}{|c}{ 15 July } & $9: 00-10: 00$ & 1 (c. 40kg) & $60+$ & $\begin{array}{l}\text { Visit 4 sky burial } \\
\text { masters }\end{array}$ \\
\hline 16 July & & 0 & 0 & $\begin{array}{l}\text { The sky burial station } \\
\text { was empty }\end{array}$ \\
\hline 17 July & & 0 & 0 & $\begin{array}{l}\text { No vultures seen on the } \\
\text { mountain }\end{array}$ \\
\hline 18 July & $8: 30-11: 00$ & $2+($ c. $80 \mathrm{~kg})$ & $130+$ & \\
\hline
\end{tabular}

Migration information from captured in the Himalayas of Bhutan, movebank and were equipped with GSM/GPS transmitters by locals with experts of From 2014 to 2016, 23 vultures were the Max Planck Institute for 
Ornithology and University of Plateau, Yunnan-Guizhou Plateau Konstanz, Germany (Sherub et al. and Mongolia-Xinjiang Plateau. 2016). The tracking data are stored at There appears to be a preference to the Movebank site congregate at garbage dumps (Figure (https://www.movebank.org) and 4), sky burial stations and slaughter have provided the first understanding houses. However, when we asked of the movements for the Himalayan four local Lamas including some sky Vulture (Sherub et al. 2016). The burial masters, they had not seen any main areas of activity for the tracked GPS transmitters on the backs of birds were in China's Qinghai-Tibet vultures.

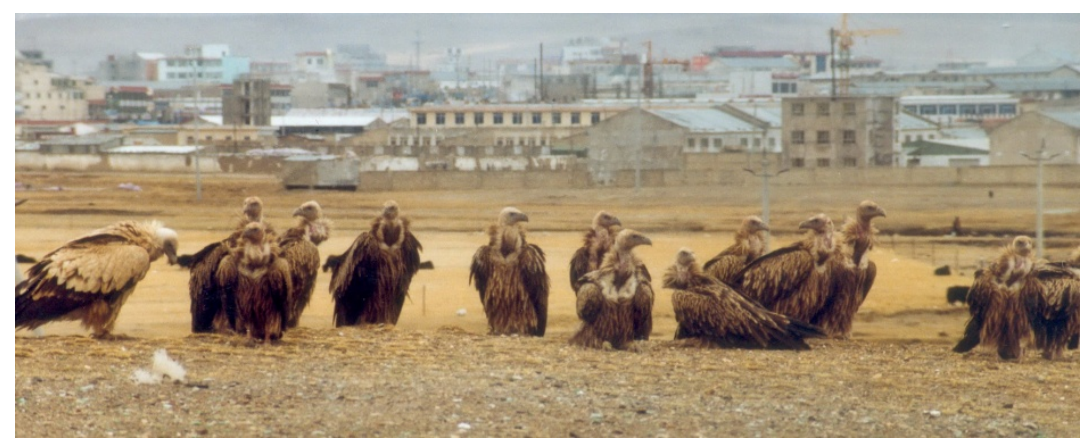

Figure 4: Vultures at a rubbish dump near a suburban area, Tibet (Photo by MaMing)

Additional data from the tagged birds have revealed that they were over-wintering from India to Nepal and Bhutan, and some in southern parts of China (Yunnan and Tibet). Tagged birds spent summers in Mongolia and China (Qinghai,
Xinjiang, Gansu, Sichuan, Inner Mongolia and Tibet). The Himalayan Vultures migrated to the summer grounds in May or June and fall migration occurred in October and November. There were two remarkable findings: First, the 
migration speed and distance. The pigeons and other species that were Himalayan Vultures flew a mean cleaning up leftover food. Such distance of about $85 \mathrm{~km}$ per day and generosity and compassion for all an annual cumulative flight distance beings are important virtues in of approximately 30,000 km! Buddhism. At the great sky burial Secondly, there was very high sites near Yushu and Sertar, Qinghai mortality recorded (> 30\% with a and Sichuan Provinces, the Tibetan GSM/GPS tag died in the first year). people have built some temples and towers of death to attract and

\section{Conservation and regulation}

At a sky burial station with a stupa in the background (Figure 5), we saw three sky burial platforms on the hillside. Next to the slopes the valley was full of prayer flags and Mani stones, crushed bones scattered over the grassland, sparrows, red-billed choughs, magpies, ravens, hill entertain the growing numbers of Chinese tourists who visit to see the vultures. It seems very surprising that sky burials are becoming an attraction for tourists, but there is no taboo (such as a ban in Tibet), which turns this simple and practical ritual into a tourist attraction in Qinghai and Sichuan. 


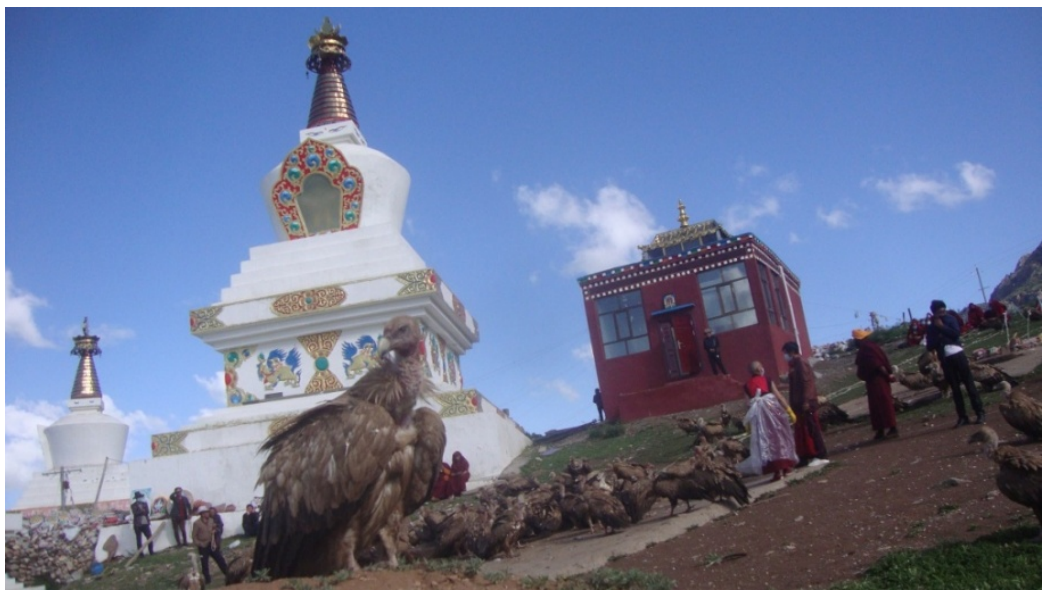

Figure 5: A sky burial station in Qinghai (Photo by MaMing)

Tibetan Buddhism plays an threats other than poisoning in China important role in the conservation of (MaMing et al. 2014).

Himalayan Vultures and other Recently, the local government wildlife in the Qinghai-Tibet Plateau passed regulations to protect Tibetan (Martin 1996, Satheesan 1998, tradition and culture. The regulations Campbell 2015). More than $80 \%$ of prohibit human activities such as the 6.28 million Tibetans are firing, blasting and quarrying around intended for eventual consumption the burial sites to avoid disturbance by Himalayan Vultures in celestial burials (MaMing et al. 2015). At one sky burial site, we were told by local people that about 100 vultures were found dead after feeding on a human carcass (Jin and Yu 2004) and, even if the local people rarely treat livestock with veterinary drugs in Tibet (Lu et al. 2009), raptors face to the scavengers. Also, the regulations do not allow sky burial for people who have died of toxicosis or infectious diseases, in an attempt to prevent poisoning of vultures. The state has recently changed its attitude toward sky burials and begun to invest heavily in the renovation of the sky burial 
stations from 2013 to 2016. Acknowledgements

Therefore, the Himalayan Vulture, a hallowed and important bird for local people and the plateau, enjoys

This research was supported by the considerable protection from several sources. We think the funeral history is an important part of Chinese history and, in the book 'Vultures in Xinjiang' (MaMing et al. 2016), we describe the story of the burial origins in relation to vulture life Xinhai.

history.

\section{References}

Anonymous. 2005. Flying with the soul of the dead. China's Tibet 16: 29-32.

Campbell, M. O. 2015. Vultures: Their evolution, ecology and conservation. CRC Press, Boca Raton.

Goss, R. E. and Klass, D.1997. Tibetan Buddhism and the resolution of grief: The Bardo-Thodol for the dying and the grieving. Death Studies 21: 377395.

Jin, Y. and Yu, Q. 2004. Vultures in the plateau. Man \& Nature 4: 74-79.

Liu, C., Huo, Z. P. and Yu, X. P. 2013. Population and conservation status of the Himalayan Griffon (Gyps himalayensis) at the Drigung Thel Monastery, Tibet, China. Chinese Birds 4: 328-331.

Lu, X., Ke, D. H., Zeng, X. H., Gong, G. and Ci, R. 2009. Status, ecology and conservation of the Himalayan griffon Gyps himalayensis (Aves, Accipitridae) in the Tibetan Plateau. Ambio 38: 166-173.

MaMing, R., Zhao X M, Xu G H, Caiwu, J., Zhang, T., Ding, P. and Xu F. 2014. Raptor conservation and culture in the west of China. Ela Journal 3: 23-29.

MaMing, R. and Guohua, Xu. 2015. Status and threats to vultures in China. Vulture News 68: 3-24.

MaMing R., Xu G H, Wu D N. 2016. Vultures in Xinjiang. Science Press, 
Beijing, China.

Martin, D.P. 1996. On the cultural ecology of sky burial on the Himalayan Plateau. East and West 46: 353-370.

National Bureau of Statistics of the People's Republic of China. 2010. http://www.stats.gov.cn/tjsj/pcsj/ Accessed November 2016

Satheesan, S.M.1998. The role of vultures in the disposal of human corpses in India and Tibet. Vulture News 39: 32-33.

Sherub, S., Wikelski, M. and Cheng, Y. C. 2016. A synoptic overview of the movement \& migration of the Himalayan Vulture in Asia. Newsletter of China Ornithological Society 25: 16-17, 40-41.

Van Dooren, T. 2011. Vulture. Reaktion Books, London.

Xinhuanet 2013. http://www.xinhuanet.com/english/ Accessed November 2016

Zhang, Y. S. 2009. The prevalence of "Bird Totem" in ancient Zhang-zhung and "Bird Burial" in Tibet. China Tibetology 7: 58-72.

Keywords: Himalayan Vulture, sky burial, Buddhism culture, jhator, tracking, Tibet

Appendix 1: Photos of a sky burial and Himalayan Vultures

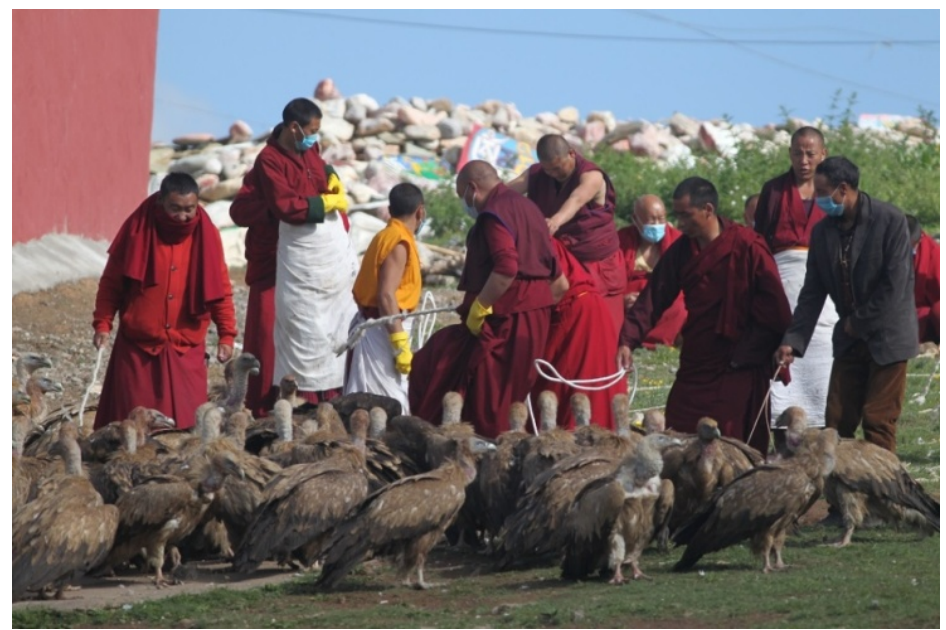

Figure A1: A sky burial with attending vultures in Qinghai (photo by MaMing) 


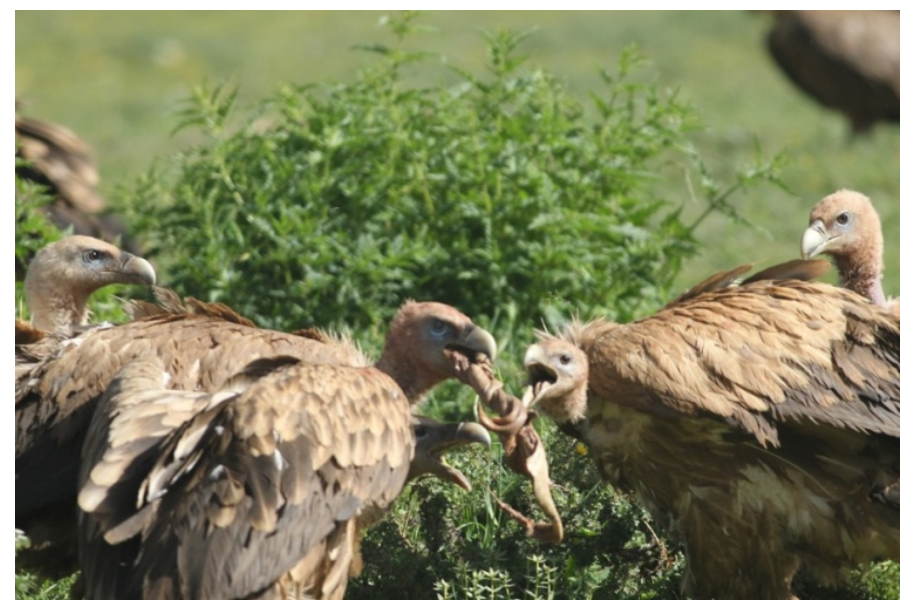

Figure A2: The vultures feeding at a sky burial site in Qinghai (Photo by MaMing)

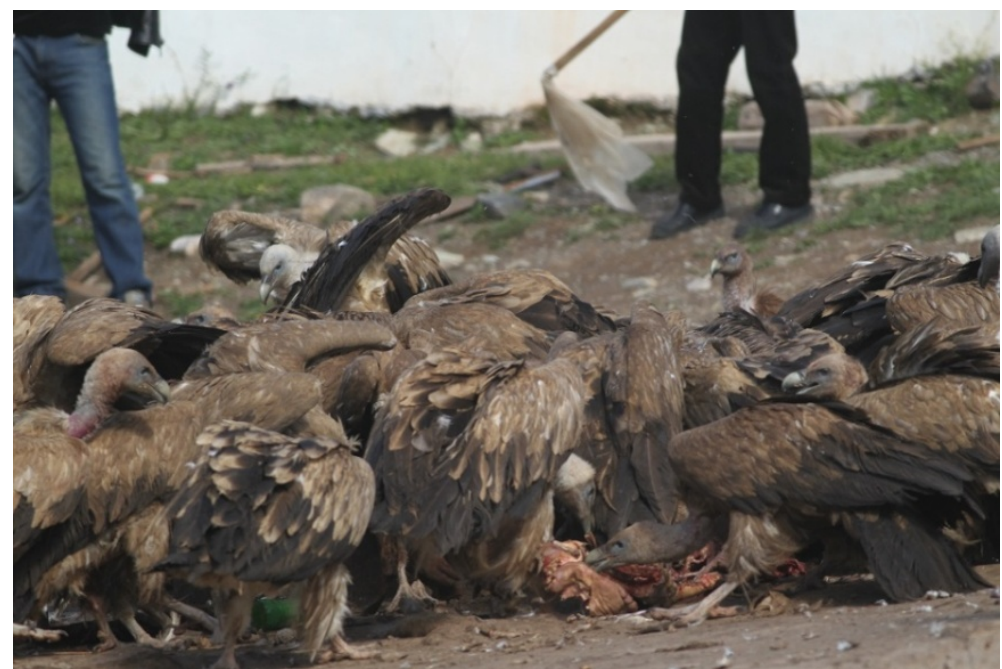

Figure A3: More than 240 vultures can finish three bodies in 2.5 hours at a sky burial site in Qinghai (Photo by MaMing) 


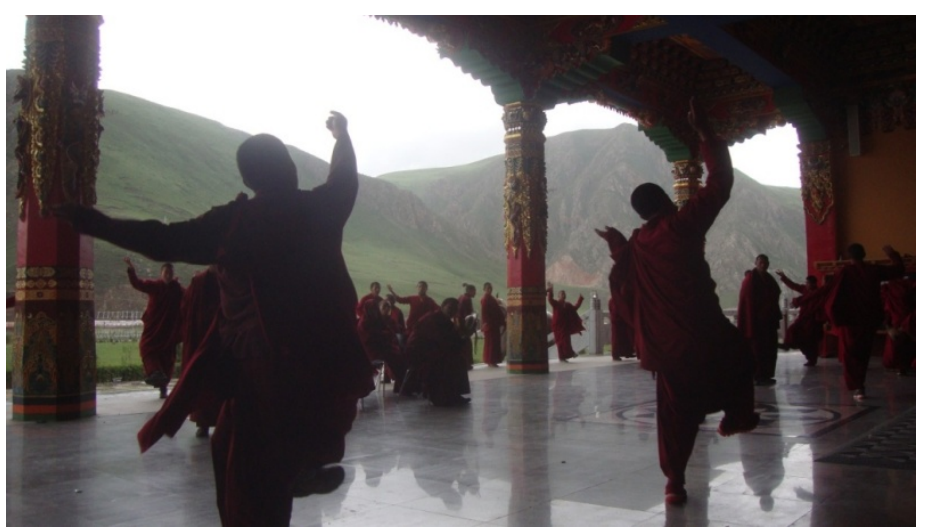

Figure A4: Lamas performing a dance like vultures (by MaMing in Qinghai)

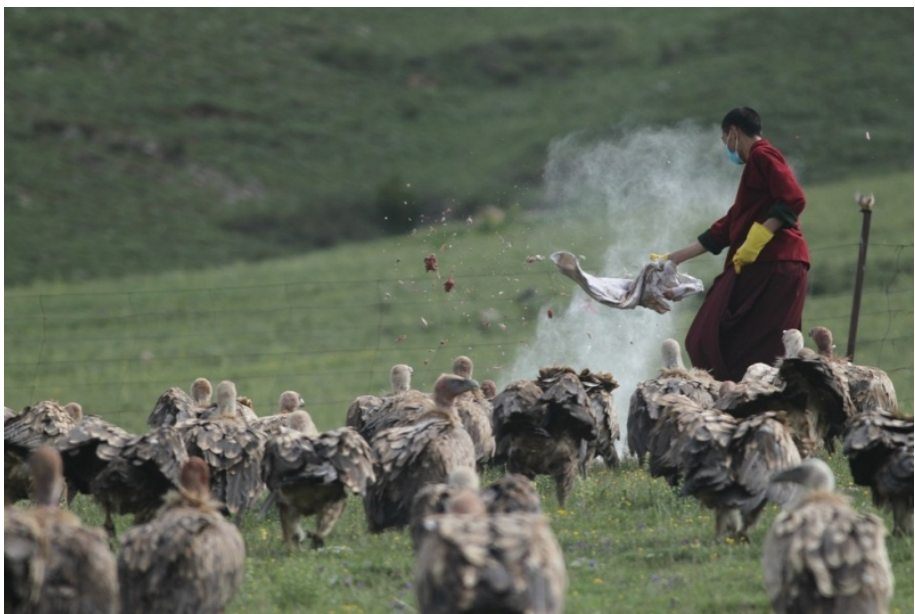

Figure A5: Vultures run to feed upon the final body parts at a sky burial in Qinghai (Photo by MaMing) 


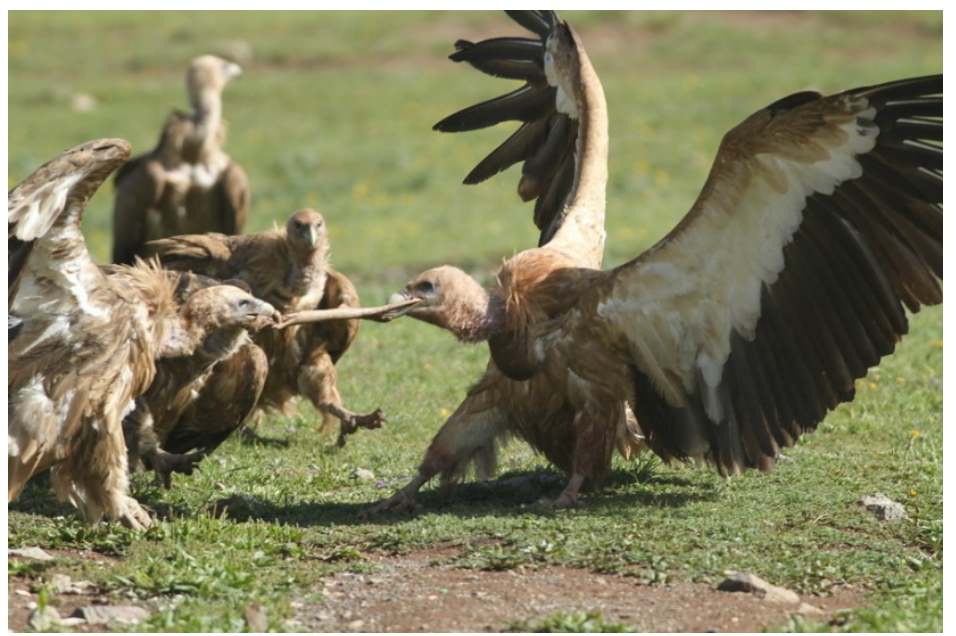

Figure A6: Vultures feeding at a sky burial site in Qinghai (Photo by MaMing)

$* * * * * *$ 